\title{
Peristaltic motion of Carreau fluid in a channel with convective boundary conditions
}

\author{
T. Hayat ${ }^{\mathrm{a}, \mathrm{b}}$, Humaira Yasmin ${ }^{\mathrm{a}, *}$ and A. Alsaedi ${ }^{\mathrm{b}}$ \\ a Department of Mathematics, Quaid-I-Azam University, Islamabad, Pakistan \\ ${ }^{\mathrm{b}}$ Department of Mathematics, Faculty of Science, King Abdulaziz University, Jeddah, Saudi Arabia
}

\begin{abstract}
We investigate the peristaltic motion of Carreau fluid in an asymmetric channel with convective boundary conditions. Mathematical formulation is first reduced in a wave frame of reference and then solutions are constructed by long wavelength and low Reynolds number conventions. Results of the stream function, axial pressure gradient, temperature and pressure rise over a wavelength are obtained for small Weissenberg number. Velocity and temperature distributions are analyzed for different parameters of interest. A comparative study between the results of Newtonian and Carreau fluids is given.
\end{abstract}

Keywords: Asymmetric channel, Carreau fluid and convective conditions

\section{Introduction}

Over the years, the dynamics of peristaltic transport of fluids has been a popular area of research. A significant amount of research articles dealing with the flows of viscous and non-Newtonian fluids in channels/tubes is reported (see few recent studies [1-13] and many refs. therein). It has been accepted that need for peristaltic pumping may arise in circumstances where it is desirable to avoid using any internal moving part such as pistons in pumping process. Such pumping generally takes place from a region of lower pressure to higher pressure. It is an inherent property of many of the smooth muscle tubes occurring in the gastrointestinal tract, in the vasomotion of small blood vessels, spermatozoa transport in the ductus efferents of the male reproductive tracts and ovum movement in the female fallopian tube. This mechanism in plant physiology is useful in phloem translocation by driving a sucrose solution along tubules by peristaltic contractions. The

*Corresponding author: Humaira Yasmin, Department of Mathematics, Quaid-I-Azam University 45320, Islamabad 44000, Pakistan. Tel.: + 9251 90642172; E-mail: qau2011@gmail.com. roller and finger pumps also operate under this principle. Interest of the recent researchers in the channel flows with heat transfer has been now substantially increased. Such interest perhaps is because of viscous developments in the electronic industry, biomedical engineering, microfabrication technologies etc. Besides these, biological tissues with heat transfer involve modes like heat conduction in tissues, heat convection by blood flow through the pores of tissue and radiation heat transfer between surface and its environment. In particular, the peristalsis with heat transfer is significant in the sense that the thermodynamic aspects of blood are useful in oxygenation and hemodialysis processes. Motivated by such facts, the peristaltic flows with heat transfer has been explored. Mention may be made to some relevant studies on the topic [14-23].

In all the above mentioned studies, the heat transfer process is addressed through prescribed boundary conditions either on temperature or heat flux. To the best of our knowledge, the convective type boundary conditions for channel walls in peristalsis are not considered yet. Therefore, the present study addresses the peristaltic transport of Carreau fluid in an asymmetric channel with convective boundary conditions. Carreau 
fluid is a type of generalized Newtonian fluid. The Carreau fluid at low shear rate behaves as a Newtonian fluid (when $n=1$ or $\Gamma=0$ ) and at high shear rate like a power-law fluid (see refs. [24] and [25]). The silicon oil is an example of Carreau fluid. The relevant equations for the fluid under consideration are first modeled and then solved. Resulting mathematical problems for long wavelength and low Reynolds number are solved. Impacts of various involved parameters in the solutions have been pointed out.

\section{Problem origination and flow equations}

We consider the problem for an incompressible Carreau fluid in a two-dimensional asymmetric channel of width $d_{1}+d_{2}$ (see Fig. 1). The $\bar{X}$ and $\bar{Y}$ axes are the rectangular coordinates with $\bar{X}$-axis along the walls of the channel and $\bar{Y}$-axis perpendicular to the $\bar{X}$-axis. The flow created is due to the imposition of the following sinusoidal waves:

$$
\begin{array}{ll}
\bar{h}_{1}(\bar{X}, \bar{t}) & \\
\quad=d_{1}+a_{1} \cos \frac{2 \pi}{\lambda}(\bar{X}-c \bar{t}), & \text { upper wall, } \\
\bar{h}_{2}(\bar{X}, \bar{t}) & \\
=-d_{2}-a_{2} \cos \left(\frac{2 \pi}{\lambda}(\bar{X}-c \bar{t})+\phi\right), & \text { lower wall. }
\end{array}
$$

In above expressions $c$ is the wave speed, $a_{1}, a_{2}$ are the waves amplitudes, $\lambda$ is the wavelength, $d_{1}+d_{2}$ is the width of the asymmetric channel, the phase difference $\phi$ varies in the range $0 \leq \phi \leq \pi(\phi=0$ corresponds to symmetric channel with waves out of phase and $\phi=\pi$ the waves are in phase) and further $a_{1}, a_{2}, d_{1}, d_{2}$ and $\phi$ satisfy the condition

$$
a_{1}^{2}+a_{2}^{2}+2 a_{1} a_{2} \cos \phi \leq\left(d_{1}+d_{2}\right)^{2} .
$$

The Carreau fluid depends upon the shear rate by the following equation

$$
\bar{\tau}=-\left[\eta_{\infty}+\left(\eta_{0}-\eta_{\infty}\right)\left(1+(\Gamma \bar{\gamma})^{2}\right)^{\frac{n-1}{2}}\right] \overline{\dot{\gamma}},
$$

where $\bar{\tau}$ is the extra stress tensor, $\eta_{\infty}$ the infinite shearrate viscosity, $\eta_{0}$ the zero shear-rate viscosity, $\Gamma$ the time constant, $n$ the dimensionless power law index and $\bar{\gamma}$ is defined as

$$
\overline{\dot{\gamma}}=\sqrt{\frac{1}{2} \sum_{i} \sum_{j} \bar{\gamma}_{i j} \bar{\gamma}_{j i}}=\sqrt{\frac{1}{2} \Pi .}
$$

Here $\Pi$ is the second invariant of strain-rate tensor. We consider the constitutive equation (3) in the case for which $\eta_{\infty}=0$, and so we can write

$$
\bar{\tau}=-\eta_{0}\left[1+(\Gamma \bar{\gamma})^{2}\right]^{\frac{n-1}{2}} \overline{\dot{\gamma}} .
$$

Note that the above model reduces to Newtonian model for $n=1$ or $\Gamma=0$. Here

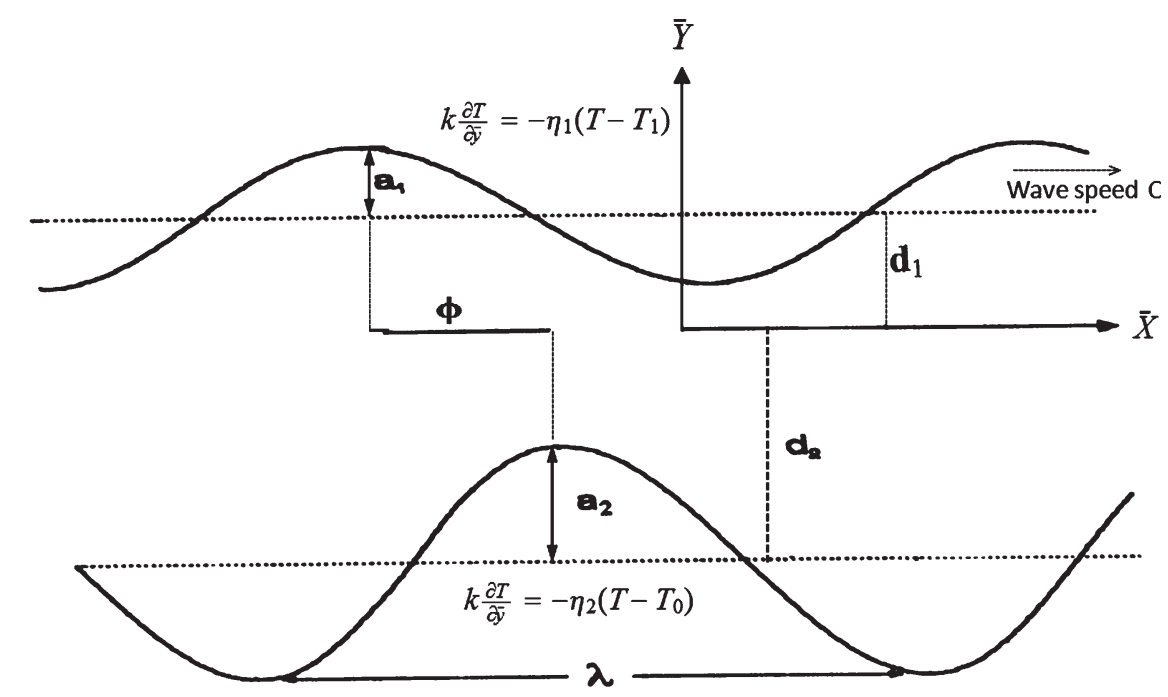

Fig. 1. Geometry of the problem. 


$$
\begin{aligned}
& \bar{\tau}_{\bar{X} \bar{X}}=-\eta_{0}\left[1+\left(\frac{n-1}{2}\right) \Gamma^{2} \overline{\dot{\gamma}}^{2}\right] \frac{\partial \bar{U}}{\partial \bar{X}}, \\
& \bar{\tau}_{\bar{X} \bar{Y}}=-\eta_{0}\left[1+\left(\frac{n-1}{2}\right) \Gamma^{2} \bar{\gamma}^{2}\right]\left(\frac{\partial \bar{U}}{\partial \bar{Y}}+\frac{\partial \bar{V}}{\partial \bar{X}}\right), \\
& \bar{\tau}_{\bar{Y} \bar{Y}}=-\eta_{0}\left[1+\left(\frac{n-1}{2}\right) \Gamma^{2} \bar{\gamma}^{2}\right] \frac{\partial \bar{V}}{\partial \bar{Y}} .
\end{aligned}
$$

Incompressibility condition is

$$
\operatorname{div} \overline{\mathbf{V}}=0
$$

The equations of motion and energy are

$$
\begin{gathered}
\rho \frac{d \overline{\mathbf{V}}}{d \bar{t}}=-\operatorname{grad} \bar{p}+\operatorname{div} \bar{\tau}, \\
\rho c_{p} \frac{d T}{d \bar{t}}=k \nabla^{2} T+\bar{\tau} \cdot(\operatorname{grad} \overline{\mathbf{V}}),
\end{gathered}
$$

in which $\overline{\mathbf{V}}$ is the velocity, $\rho$ the density of fluid, $\frac{d}{d \bar{t}}$ the material time derivative, $T$ the fluid temperature, $c_{p}$ the specific heat, $k$ the thermal conductivity of the material, the Cauchy stress tensor $\bar{\tau}, \bar{p}$ the pressure and $\nabla^{2}=$ $\left(\frac{\partial^{2}}{\partial \bar{X}^{2}}+\frac{\partial^{2}}{\partial \bar{Y}^{2}}\right)$ (The over bar refers to a dimensional quantity).

The exchange of heat at the walls is given by

$$
\begin{aligned}
& k \frac{\partial T}{\partial \bar{Y}}=-\eta_{1}\left(T-T_{1}\right) \text { at } \bar{Y}=\bar{h}_{1}, \\
& k \frac{\partial T}{\partial \bar{Y}}=-\eta_{2}\left(T-T_{0}\right) \text { at } \bar{Y}=\bar{h}_{2},
\end{aligned}
$$

where $\eta_{1}$ and $\eta_{2}, T_{1}$ and $T_{0}$ are the heat transfer coefficients and the temperatures at the upper and lower channel walls respectively.

For two-dimensional flow of Carreau fluid, we have the velocity $\overline{\mathbf{V}}$ in the form

$$
\overline{\mathbf{V}}=(\bar{U}(\bar{X}, \bar{Y}, \bar{t}), \bar{V}(\bar{X}, \bar{Y}, \bar{t}), 0) .
$$

In the laboratory frame $(\bar{X}, \bar{Y})$ the flow is unsteady. However if observed in a coordinate system moving at the wave speed $c$ (wave frame) $(\bar{x}, \bar{y})$ it can be treated as steady. The coordinates and velocities in the two frames are

$$
\begin{aligned}
& \bar{x}=\bar{X}-c \bar{t}, \bar{y}=\bar{Y}, \bar{u}(\bar{x}, \bar{y})=\bar{U}(\bar{X}, \bar{Y}, \bar{t})-c, \\
& \bar{v}(\bar{x}, \bar{y})=\bar{V}(\bar{X}, \bar{Y}, \bar{t}), T(\bar{x}, \bar{y})=T(\bar{X}, \bar{Y}, \bar{t}),
\end{aligned}
$$

where $\bar{u}$ and $\bar{v}$ indicate the velocity components in the wave frame.
Introducing

$$
\begin{aligned}
& x=\frac{\bar{x}}{\lambda}, y=\frac{\bar{y}}{d_{1}}, u=\frac{\bar{u}}{c}, v=\frac{\bar{v}}{c \delta}, t=\frac{c}{\lambda} \bar{t}, \\
& p=\frac{d_{1}^{2} \bar{p}}{c \lambda \eta_{0}}, W e=\frac{\Gamma c}{d_{1}}, h_{1}=\frac{\bar{h}_{1}}{d_{1}}, h_{2}=\frac{\bar{h}_{2}}{d_{1}}, t=\frac{c \bar{t}}{\lambda}, \\
& \tau_{x x}=\frac{\lambda}{c \eta_{0}} \bar{\tau}_{\bar{x} \bar{x}}, \tau_{x y}=\frac{d_{1}}{c \eta_{0}} \bar{\tau}_{\bar{x} \bar{y}}, \dot{\gamma}=\frac{\bar{\gamma} d_{1}}{c}, \tau_{y y}=\frac{d_{1}}{c \eta_{0}} \bar{\tau}_{\bar{y} \bar{y}},
\end{aligned}
$$$$
\theta=\frac{T-T_{0}}{T_{1}-T_{0}}, \delta=\frac{d_{1}}{\lambda}, \operatorname{Re}=\frac{\rho c d_{1}}{\eta_{0}}, \operatorname{Pr}=\frac{\mu c_{p}}{k},
$$

$$
E c=\frac{c^{2}}{\left(T_{1}-T_{0}\right) c_{p}},
$$

along with Eq. (12) and defining the stream function $\psi(x, y)$ by

$$
u=\frac{\partial \psi}{\partial y}, v=-\frac{\partial \psi}{\partial x},
$$

equation (6) is satisfied identically and Eqs. (7) and (8) yield

$$
\begin{aligned}
\delta \operatorname{Re} & {\left[\left(\frac{\partial \psi}{\partial y} \frac{\partial}{\partial x}-\frac{\partial \psi}{\partial x} \frac{\partial}{\partial y}\right)\left(\frac{\partial \psi}{\partial y}\right)\right]+\frac{\partial p}{\partial x} } \\
& =-\delta^{2} \frac{\partial \tau_{x x}}{\partial x}-\frac{\partial \tau_{x y}}{\partial y}, \\
-\delta^{3} \operatorname{Re} & {\left[\left(\frac{\partial \psi}{\partial y} \frac{\partial}{\partial x}-\frac{\partial \psi}{\partial x} \frac{\partial}{\partial y}\right)\left(\frac{\partial \psi}{\partial x}\right)\right]+\frac{\partial p}{\partial y} } \\
= & -\delta^{2} \frac{\partial \tau_{x y}}{\partial x}-\delta \frac{\partial \tau_{y y}}{\partial y},
\end{aligned}
$$

$$
\begin{aligned}
\delta \operatorname{Re}\left[\frac{\partial \psi}{\partial y} \frac{\partial}{\partial x}-\frac{\partial \psi}{\partial x} \frac{\partial}{\partial y}\right] \theta & =\frac{1}{\operatorname{Pr}}\left(\delta^{2} \frac{\partial^{2}}{\partial x^{2}}+\frac{\partial^{2}}{\partial y^{2}}\right) \theta+E c\left[\delta \frac{\partial^{2} \psi}{\partial x \partial y}\left(\tau_{x x}-\tau_{y y}\right)\right. \\
& \left.+\left(\frac{\partial^{2} \psi}{\partial y^{2}}-\delta^{2} \frac{\partial^{2} \psi}{\partial x^{2}}\right) \tau_{x y}\right]
\end{aligned}
$$

where the components of extra stress tensor from Eq. (5) are

$$
\begin{gathered}
\tau_{x x}=-2\left[1+\frac{n-1}{2} W e^{2} \dot{\gamma}^{2}\right] \frac{\partial^{2} \psi}{\partial x \partial y}, \\
\tau_{x y}=-\left[1+\frac{n-1}{2} W e^{2} \dot{\gamma}^{2}\right]\left(\frac{\partial^{2} \psi}{\partial y^{2}}-\delta^{2} \frac{\partial^{2} \psi}{\partial x^{2}}\right),
\end{gathered}
$$




$$
\begin{gathered}
\tau_{y y}=2 \delta\left[1+\frac{n-1}{2} W e^{2} \dot{\gamma}^{2}\right] \frac{\partial^{2} \psi}{\partial x \partial y}, \\
\dot{\gamma}=\left[2 \delta^{2}\left(\frac{\partial^{2} \psi}{\partial x \partial y}\right)^{2}+\left(\frac{\partial^{2} \psi}{\partial y^{2}}-\delta^{2} \frac{\partial^{2} \psi}{\partial x^{2}}\right)^{2}\right. \\
\left.+2 \delta^{2}\left(\frac{\partial^{2} \psi}{\partial x \partial y}\right)^{2}\right]^{\frac{1}{2}} .
\end{gathered}
$$

$$
\begin{gathered}
\frac{\partial p}{\partial x}=\frac{\partial}{\partial y}\left[\left(1+\frac{n-1}{2} W e^{2}\left(\frac{\partial^{2} \psi}{\partial y^{2}}\right)^{2}\right) \frac{\partial^{2} \psi}{\partial y^{2}}\right] \\
\frac{\partial p}{\partial y}=0
\end{gathered}
$$

The above equation indicates that $p \neq p(y)$. Eliminating the pressure $p$ from Eqs. (24) and (25) we get the governing equation for the stream function as

$$
\frac{\partial^{2}}{\partial y^{2}}\left[\left(1+\frac{n-1}{2} W e^{2}\left(\frac{\partial^{2} \psi}{\partial y^{2}}\right)^{2}\right) \frac{\partial^{2} \psi}{\partial y^{2}}\right]=0 .
$$

Weissenberg, Prandtl and Eckert numbers respectively. Equations (9) and (10) give

$$
\begin{aligned}
\frac{\partial \theta}{\partial y}+B i_{1}(\theta-1) & =0 \text { at } y=h_{1}, \\
\frac{\partial \theta}{\partial y}+B i_{2} \theta & =0 \text { at } y=h_{2},
\end{aligned}
$$

where $B i_{1}=\eta_{1} d_{1} / k$ and $B i_{2}=\eta_{2} d_{1} / k$ are the Biot numbers.

In the limit $\operatorname{Re} \rightarrow 0$, the inertialess flow corresponds to Poiseuille-like longitudinal velocity profile. The pressure gradient depends upon $x$ and $t$ only in laboratory frame. It does not depend on $y$. Such features can be expected because there is no streamline curvature to produce transverse pressure gradient when $\delta=0$. The assumptions of long wavelength and small Reynolds number gives $\delta=0$ and $\operatorname{Re}=0$. It should be pointed out that the theory of long wavelength and zero Reynolds number remains applicable for case of chyme transport in male small intestine [26]. In this case $c=2 \mathrm{~cm} / \mathrm{min}, a=1.25 \mathrm{~cm}$ and $\lambda=8.01 \mathrm{~cm}$. Here half width of intestine is small in comparison to wavelength. i.e. $a / \lambda=0.156$. It is also declared by Lew et al. [27] that Reynolds number in small intestine was small. Further, the situation of intrauterine fluid flow due to myometrial contractions is a peristaltic type fluid motion in a cavity. The sagittal cross section of the uterus reveals a narrow channel enclosed by two fairly parallel walls [28]. The $1-3 \mathrm{~mm}$ width of this channel is very small compared with its $50 \mathrm{~mm}$ length [29], defining an opening angle from cervix to fundus of about 0.04 rad. Analysis of dynamics parameters of the uterus revealed frequency, wavelength, amplitude and velocity of the fluid-wall interface during a typical contractile wave were found to be $0.01-0.057$ $\mathrm{Hz}, 10-30 \mathrm{~mm}, 0.05-0.2 \mathrm{~mm}$ and $0.5-1.9 \mathrm{~mm} / \mathrm{s}$ respectively. Therefore under the assumption of long wavelength $\delta \ll 1$ and low Reynolds number $\operatorname{Re} \rightarrow 0$, Eqs. (15) and (16) reduce to
Also Eq. (17) reduces to

$$
\frac{\partial^{2} \theta}{\partial y^{2}}+B r\left(\frac{\partial^{2} \psi}{\partial y^{2}}\right)^{2}\left[1+\frac{n-1}{2} W e^{2}\left(\frac{\partial^{2} \psi}{\partial y^{2}}\right)^{2}\right]=0,
$$

where the Brinkman number

$$
B r=\operatorname{Pr} E c,
$$

In the fixed frame, the instantaneous volume flow rate is given by

$$
Q=\int_{\bar{h}_{2}(\bar{X}, \bar{t})}^{\bar{h}_{1}(\bar{X}, \bar{U})} \bar{U}(\bar{X}, \bar{Y}, \bar{t}) d \bar{Y} .
$$

The volume flow rate in wave frame is

$$
q=\int_{\bar{h}_{2}(\bar{x})}^{\bar{h}_{1}(\bar{x})} \bar{u}(\bar{x}, \bar{y}) d \bar{y} .
$$

From Eqs. (12), (29) and (30) we can write

$$
Q=q+c \bar{h}_{1}(\bar{x})-c \bar{h}_{2}(\bar{x}) .
$$

The time-mean flow over a period $L$ is defined as

$$
\bar{Q}=\frac{1}{L} \int_{0}^{L} Q d \bar{t} .
$$

Substituting Eq. (31) into Eq. (32) and then integrating the resulting expression one has

$$
\bar{Q}=q+c d_{1}+c d_{2} .
$$

Defining $\Theta$ and $F$ as the dimensionless time-mean flows in the laboratory and wave frames respectively by

$$
\Theta=\frac{\bar{Q}}{c d_{1}}, F=\frac{q}{c d_{1}},
$$

equation (33) becomes 


$$
\Theta=F+1+d
$$

with

$$
F=\int_{h_{2}(x)}^{h_{1}(x)} \frac{\partial \psi}{\partial y} d y=\psi\left(h_{1}(x)\right)-\psi\left(h_{2}(x)\right) .
$$

The dimensionless forms of $h_{i}(i=1,2)$ are

$$
\begin{aligned}
& h_{1}(x)=1+a \cos (2 \pi x), \\
& h_{2}(x)=-d-b \cos (2 \pi x+\phi),
\end{aligned}
$$

where $a=a_{1} / d_{1}, b=a_{2} / d_{1}, d=d_{2} / d_{1}$ and $\phi$ satisfies the following relation

$$
a^{2}+b^{2}+2 a b \cos \phi \leq(1+d)^{2} .
$$

The conditions for the dimensionless stream function in wave frame are

$$
\begin{aligned}
& \psi=\frac{F}{2}, \quad \frac{\partial \psi}{\partial y}=-1, \quad \text { at } y=h_{1}(x), \\
& \psi=-\frac{F}{2}, \quad \frac{\partial \psi}{\partial y}=-1, \quad \text { at } y=h_{2}(x) .
\end{aligned}
$$

\section{Perturbation solution}

For perturbation solution, we expand $\psi, \theta, \mathrm{d} p / d x$ and $F$ as follows:

$$
\begin{aligned}
\psi & =\psi_{0}+W e^{2} \psi_{1}+O\left(W e^{4}\right), \\
\theta & =\theta_{0}+W e^{2} \theta_{1}+O\left(W e^{4}\right), \\
d p / d x & =d p_{0} / d x+W e^{2}\left(d p_{1} / d x\right)+O\left(W e^{4}\right), \\
F & =F_{0}+W e^{2} F_{1}+O\left(W e^{4}\right) .
\end{aligned}
$$

Substitution of above equations into Eqs. (22-24), (26), (27), (39), (40) and then collecting the terms of like powers of $W e^{2}$ yield:

\subsection{System of order $W e^{0}$}

$$
\begin{aligned}
& \frac{\partial^{4} \psi_{0}}{\partial y^{4}}=0, \\
& \frac{\partial^{2} \theta_{0}}{\partial y^{2}}+B r\left(\frac{\partial^{2} \psi_{0}}{\partial y^{2}}\right)^{2}=0, \\
& \frac{d p_{0}}{d x}=\frac{\partial^{3} \psi_{0}}{\partial y^{3}},
\end{aligned}
$$

$\psi_{0}=\frac{F_{0}}{2}, \frac{\partial \psi_{0}}{\partial y}=-1, \frac{\partial \theta_{0}}{\partial y}+B i_{1}\left(\theta_{0}-1\right)=0$,

$$
\text { at } y=h_{1}(x) \text {, }
$$

$$
\psi_{0}=\frac{-F_{0}}{2}, \frac{\partial \psi_{0}}{\partial y}=-1, \frac{\partial \theta_{0}}{\partial y}+B i_{2} \theta_{0}=0,
$$

$$
\text { at } y=h_{2}(x) \text {. }
$$

\subsection{System of order $W e^{2}$}

$$
\begin{gathered}
\frac{\partial^{4} \psi_{1}}{\partial y^{4}}+\left(\frac{n-1}{2}\right) \frac{\partial^{2}}{\partial y^{2}}\left(\frac{\partial^{2} \psi_{0}}{\partial y^{2}}\right)^{3}=0, \\
\frac{\partial^{2} \theta_{1}}{\partial y^{2}}+B r\left[2\left(\frac{\partial^{2} \psi_{0}}{\partial y^{2}}\right)\left(\frac{\partial^{2} \psi_{1}}{\partial y^{2}}\right)\right. \\
\left.+\left(\frac{n-1}{2}\right)\left(\frac{\partial^{2} \psi_{0}}{\partial y^{2}}\right)^{4}\right]=0, \\
\frac{d p_{1}}{d x}=\frac{\partial^{3} \psi_{1}}{\partial y^{3}+\left(\frac{n-1}{2}\right) \frac{\partial}{\partial y}\left(\frac{\partial^{2} \psi_{0}}{\partial y^{2}}\right)^{3},} \\
\psi_{1}=\frac{F_{1}}{2}, \frac{\partial \psi_{1}}{\partial y}=0, \frac{\partial \theta_{1}}{\partial y}+B i_{1} \theta_{1}=0,
\end{gathered}
$$

at $y=h_{1}(x)$,

$$
\psi_{1}=\frac{-F_{1}}{2}, \frac{\partial \psi_{1}}{\partial y}=0, \frac{\partial \theta_{1}}{\partial y}+B i_{2} \theta_{1}=0,
$$

$$
\text { at } y=h_{2}(x) \text {. }
$$

\subsection{Solution for system of order $W e^{0}$}

The solutions of the Eqs. (41) and (42) subject to the boundary conditions (44) and (45) are

$$
\begin{aligned}
& \psi_{0}=R_{1} y^{3}+R_{2} y^{2}+R_{3} y+R_{4}, \\
& \theta_{0}=A_{1} y^{4}+A_{2} y^{3}+A_{3} y^{2}+A_{4} y+A_{5},
\end{aligned}
$$

where the values of $R_{i}(i=1-4)$ and $A_{j}(j=1-5)$ appearing in above equations are given in Appendix A.

The longitudinal velocity and pressure gradient are given by

$$
\begin{aligned}
u_{0} & =3 R_{1} y^{2}+2 R_{2} y+R_{3}, \\
\frac{d p_{0}}{d x} & =6 R_{1} .
\end{aligned}
$$

The non-dimensional pressure rise per wavelength $\left(\Delta P_{\lambda_{0}}\right)$ is given by 


$$
\Delta P_{\lambda_{0}}=\int_{0}^{1} \frac{d p_{0}}{d x} d x .
$$

We note that the solution expressions at this order are for the Newtonian fluid.

\subsection{Solution for system of order $W e^{2}$}

Substituting Eq. (51) into Eqs. (46-48), solving the resulting equations and then applying the corresponding boundary conditions we get the solutions for $\psi_{1}$, $u_{1}, d p_{1} / d x$ and $\theta_{1}$ in the forms as follows:

$$
\begin{aligned}
\psi_{1}= & M_{1} y^{5}+M_{2} y^{4}+M_{3} y^{3}+M_{4} y^{2} \\
& +M_{5} y+M_{6}, \\
u_{1}= & 5 M_{1} y^{4}+4 M_{2} y^{3}+3 M_{3} y^{2}+2 M_{4} y+M_{5},
\end{aligned}
$$

$$
\begin{aligned}
\frac{d p_{1}}{d x}= & -\frac{2}{5\left(h_{1}-h_{2}\right)^{3}}\left[30 F_{1}-\left(h_{1}-h_{2}\right)^{3}(-1+n)\right. \\
& \left(3\left(h_{1}+h_{2}\right) R_{1}+2 R_{2}\right) \times\left(9 \left(2 h_{1}^{2}+h_{1} h_{2}\right.\right. \\
& \left.\left.\left.+2 h_{2}^{2}\right) R_{1}^{2}+15\left(h_{1}+h_{2}\right) R_{1} R_{2}+5 R_{2}^{2}\right)\right],(58) \\
\theta_{1}= & L_{1} y^{6}+L_{2} y^{5}+L_{3} y^{4}+L_{4} y^{3} \\
& +L_{5} y^{2}+L_{6} y+L_{7},
\end{aligned}
$$

where the values of $M$ 's and $L$ 's in above equations are given in Appendix A. The pressure rise per wavelength $\left(\Delta P_{\lambda_{1}}\right)$ is given by

$$
\Delta P_{\lambda_{1}}=\int_{0}^{2 \pi} \frac{d p_{1}}{d x} d x .
$$

The perturbation expressions of $\psi, \theta, \Delta P_{\lambda}$ and $d p / d x$ upto $W e^{2}$ are

$$
\begin{aligned}
\psi & =\psi_{0}+W e^{2} \psi_{1} . \\
\theta & =\theta_{0}+W e^{2} \theta_{1} . \\
\frac{d p}{d x} & =\frac{d p_{0}}{d x}+W e^{2} \frac{d p_{1}}{d x} . \\
\Delta P_{\lambda} & =\Delta P_{\lambda_{0}}+W e^{2} \Delta P_{\lambda_{1}} .
\end{aligned}
$$

\section{Results and discussion}

To discuss qualitatively the behavior of embedding parameters of interest on flow quantities such as longitudinal velocity $u$ and temperature distribution $\theta$, we have prepared Figures 2-10. The effects of various parameters on pressure gradient $d p / d x$, pressure rise per wavelength $\Delta p_{\lambda}$ and pumping and trapping are already investigated by Ali and Hayat [3]. Hence we avoid to include such results here. However the longitudinal velocity for different values of $W e, n, \phi$ and $\Theta$ have been plotted in Figures 2-5. Effects of We and $n$ on the longitudinal velocity are indicated in the Figures 2 and 3. These Figures depict that velocity for Newtonian fluid (when $W e=0.0$ and $n=1$ ) is greater than the Carreau fluid (when $W e=0.3,0.4$ and $n=0.398$, 0.496) in the narrow part of the channel. But in wider part of the channel we witness the opposite behavior. Actually the Weissenberg number (having the time constant) indicates the degree of anisotropy or orientation generated by the deformation, and is appropriate to describe flows with a constant stretch history, such as simple shear. Fig. 4 designates the effect of phase angle



Fig. 2. Plot showing $u$ versus $y$. Here $a=0.6, b=0.3, d=1.1$, $\Theta=4, \phi=\pi / 6, x=0.25$ and $n=0.398$.



Fig. 3. Plot showing $u$ versus $y$. Here $a=0.6, b=0.3, d=1.1$, $\Theta=4, \phi=\pi / 6, x=0.25$ and $W e=0.4$. 




Fig. 4. Plot showing $u$ versus $y$. Here $a=0.6, b=0.3, d=1.1$, $\Theta=4, n=0.398, x=0.25$ and $W e=0.4$.

$\phi$ on longitudinal velocity $u$. This Fig. directs to the result that $u$ decreases when $\phi$ increases in lower part of the channel and such behavior is quite opposite in the other region. This is because of the presence of phase difference in the geometry of the lower wall of asymmetric channel. In Fig. 5, the effect of $\Theta$ on longitudinal velocity is shown. It is perceived that with an increase in $\Theta$, the velocity increases. The flow rate is directly proportional to the velocity of the fluid. Fig. 6 confirms an increase in fluid temperature with maximum value along the centerline and minimum at the walls due to convective conditions. It is interesting to note that the fluid temperature generally increases with increasing values of the Brinkman number $\mathrm{Br}$. Actually Brinkman number $\mathrm{Br}$ involves viscous dissipation effects which are due to the energy production and thus temperature enhances. Fig. 7 discloses that by increasing the value of $B i_{1}$ the temperature profile $\theta$ decreases at the upper wall while it has no significant effect near the lower wall of channel. Also the temperature $\theta$ increases near the lower wall by increasing the Biot number $B i_{2}$ and it has no effect on temperature profile near the upper wall of the channel (see Fig. 8). Here we have considered the values of Biot numbers much larger than 0.1 due to non-uniform temperature fields within the fluid. However problems involving small Biot numbers are thermally simple due to the uniform temperature fields within the fluid. It is important to mention that for larger values of Biot numbers the results for the prescribed surface temperature are obtained as a special case of the present problem. Fig. 9 portrays the temperature distribution for numerous values of $W e$. It is found that the temperature distribution is larger for the Newtonian fluid $(W e=0)$ when compared with

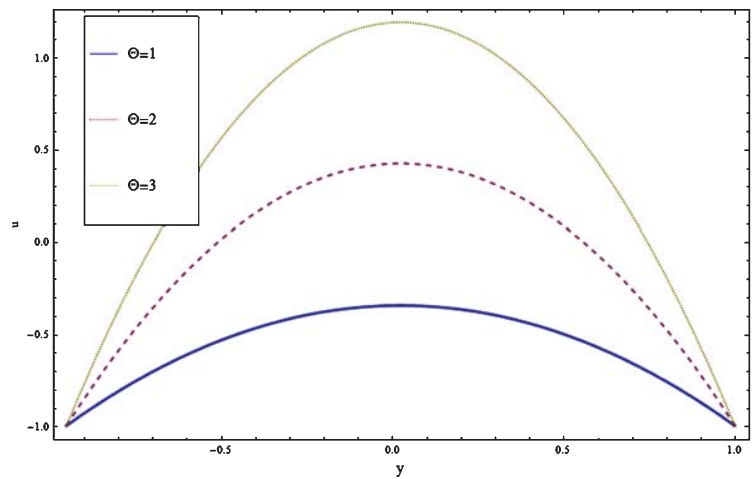

Fig. 5. Plot showing $u$ versus $y$. Here $a=0.6, b=0.3, d=1.1$, $\phi=\pi / 6, n=0.398, x=0.25$ and $W e=0.4$.

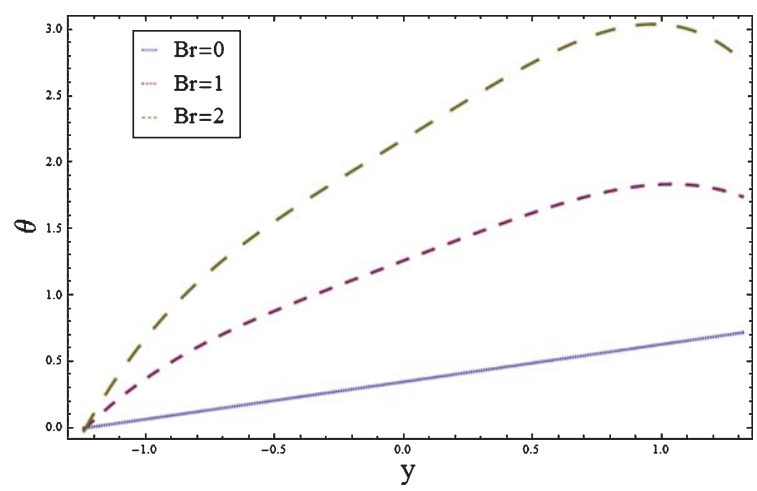

Fig. 6. Plot showing $\theta$ versus $y$. Here $a=0.3, b=0.5, d=1, \phi=$ $\pi / 4, \Theta=1.5, n=0.398, B i_{1}=1, B i_{2}=10, x=0.2$ and $W e=$ 0.1

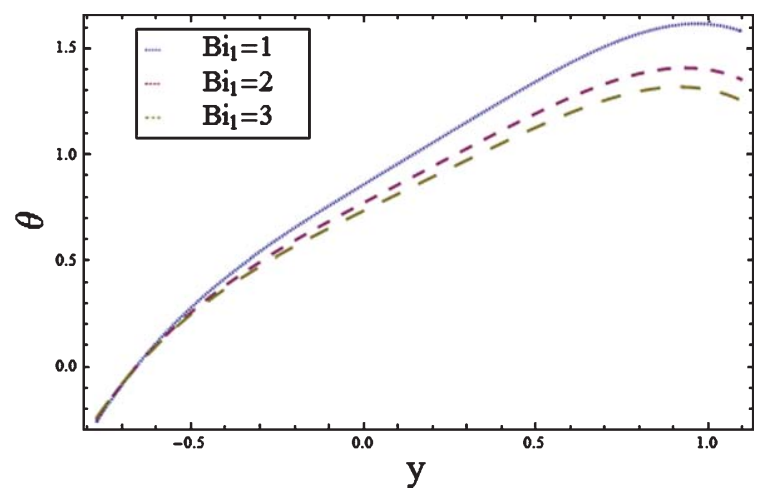

Fig. 7. Plot showing $\theta$ versus $y$. Here $a=0.3, b=0.5, d=1, \phi=$ $\pi / 4, \Theta=1.5, n=0.398, B r=1, B i_{2}=10, x=0.2$ and $W e=0.3$.

the Carreau fluid ( $W e=0.2,0.4$ ). Similar behavior is observed for the power-law index $n$ in Fig. 10. Here for $n=1$ (Newtonian fluid), the temperature distribu- 




Fig. 8. Plot showing $\theta$ versus $y$. Here $a=0.3, b=0.5, d=1, \phi=$ $\pi / 4, \Theta=1.5, n=0.398, B i_{1}=10, B r=1, x=0.2$ and $W e=0.3$.

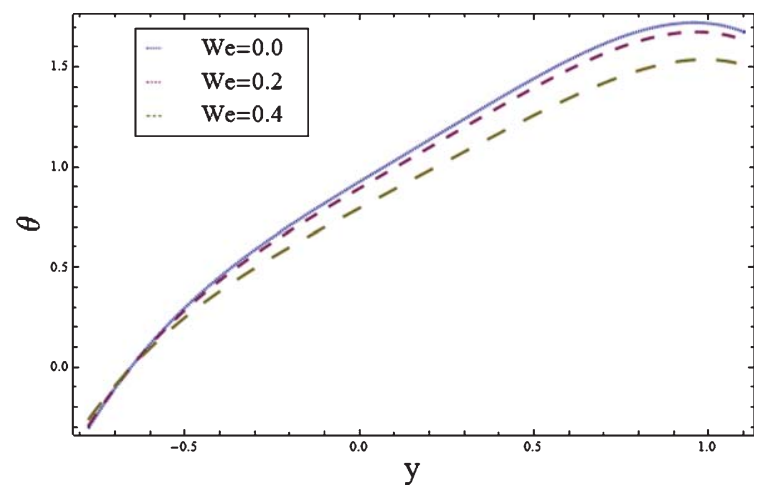

Fig. 9. Plot showing $\theta$ versus $y$. Here $a=0.3, b=0.5, d=1, \phi=$ $\pi / 4, \Theta=1.5, n=0.398, B i_{1}=1, B i_{2}=10, x=0.2$ and $B r=1$.

tion is greater than the Carreau fluid (when $n=0.398$, 0.496). The behaviors of parameters on the heat transfer coefficient at the upper wall have been observed in the Figures 11-14. The heat transfer coefficient is denoted by $Z(x)=\left(h_{1}\right)_{x} \theta_{y}\left(h_{1}\right)$ which actually defines the rate of heat transfer or heat flux at the upper wall. The heat transfer coefficient $Z(x)$ increases when the values of Brinkman number $\mathrm{Br}$ are set to be increased (see Fig. 11). Here $B r=0$ shows the absence of viscous dissipation effects. It is also noteworthy that heat transfer coefficient $Z(x)$ is higher when we consider the viscous dissipation effects (i.e. when $\mathrm{Br} \neq 0$ ). Further the behavior of heat transfer coefficient is oscillatory due to the propagation of sinusoidal waves along the channel walls. According to Fig. 12 the heat transfer coefficient $Z(x)$ decreases by increasing $W e$. Also heat transfer coefficient $Z(x)$ is higher for Newtonian fluid $(W e=0)$ than the Carreau fluid $(W e \neq 0)$. It is inter-

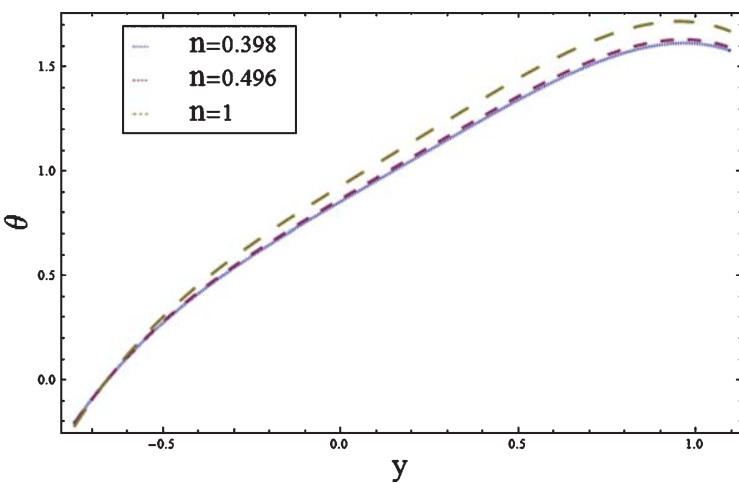

Fig. 10. Plot showing $\theta$ versus $y$. Here $a=0.3, b=0.5, d=1, \phi=$ $\pi / 4, \Theta=1.5, B r=1, B i_{1}=1, B i_{2}=10, x=0.2$ and $W e=0.1$.



Fig. 11. Plot showing $Z(x)$ versus $x$. Here $a=0.3, b=0.5, d=1$, $\phi=\pi / 4, \Theta=1.5, B i_{1}=1, B i_{2}=10, n=0.398$ and $W e=0.1$.

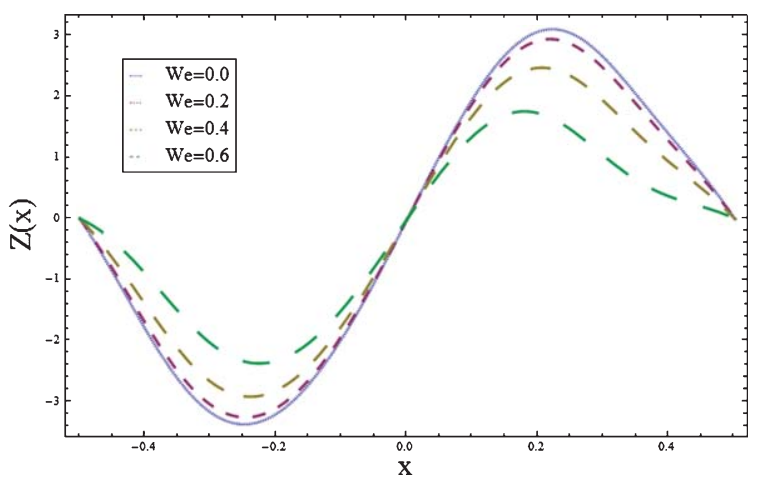

Fig. 12. Plot showing $Z(x)$ versus $x$. Here $a=0.3, b=0.5, d=1$, $\phi=\pi / 4, \Theta=1.5, B r=2, B i_{1}=1, B i_{2}=10$ and $n=0.398$.

esting to note that the heat transfer coefficient $Z(x)$ increases for increasing values of Biot number $B i_{1}$ and $B i_{2}$ (see Figures 13 and 14). 


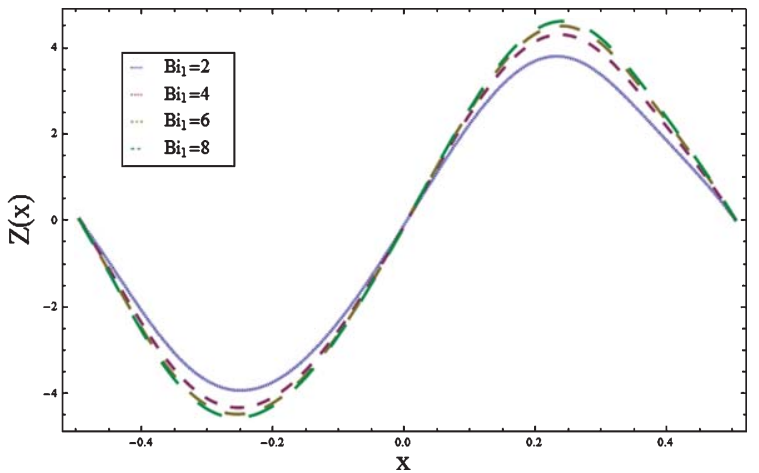

Fig. 13. Plot showing $Z(x)$ versus $x$. Here $a=0.3, b=0.5, d=1$, $\phi=\pi / 4, \Theta=1.5, B r=2, B i_{2}=10, n=0.398$ and $W e=0.1$.

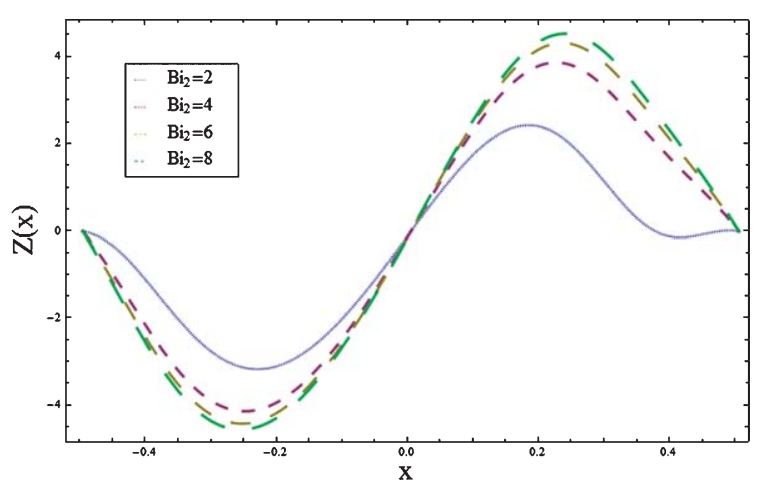

Fig. 14. Plot showing $Z(x)$ versus $x$. Here $a=0.3, b=0.5, d=1$, $\phi=\pi / 4, \Theta=1.5, B r=2, B i_{1}=10, n=0.398$ and $W e=0.1$.

\section{Concluding remarks}

A mathematical model subject to long wavelength and low Reynolds number approximations is presented in order to study the effects of convective boundary conditions on peristaltic transport of Carreau fluid in an asymmetric channel. Series expressions of stream function, longitudinal velocity and pressure gradient are developed. A comparative study is made between the Newtonian and Carreau fluids. It is concluded that at the center of channel, the longitudinal velocity for Newtonian fluid is greater than the Carreau fluid while near the walls of the channel the velocity for Newtonian fluid is lower than that of a Carreau fluid. The thermal study discloses that an increase in Biot number at the lower wall $\mathrm{Bi}_{2}$ and $\mathrm{Br}$ boosts the fluid temperature while it decreases for the Biot number at the upper wall of the channel $B i_{1}$. It is also interesting to note that for larger values of Biot numbers, the results for the prescribed surface temperature are obtained as a special case of the present study. Also the fluid temperature for a Newtonian fluid is higher than Carreau fluid. The heat transfer coefficient at the upper wall is an increasing function of Biot numbers.

\section{Acknowledgment}

The useful suggestions of the reviewers about its earlier version are greatly appreciated. Further, the first two authors are grateful to the Higher Education Commision (HEC) of Pakistan for the financial support through PhD Indigenous Fellowship Program.

\section{References}

[1] Kh.S. Mekheimer, Non-linear peristaltic transport of a secondgrade fluid through a porous medium, Appl Math Model 35 (2011), 2695.

[2] S. Srinivas and V. Pushparaj, Non-linear peristaltic transport in an inclined asymmetric channel, Comm Nonlinear Sci Numer Simul 13 (2008), 1782.

[3] N. Ali and T. Hayat, Peristaltic motion of a Carreau fluid in an asymmetric channel, Appl Math and Comput 193 (2007), 535.

[4] D. Tripathi, Numerical study on peristaltic transport of fractional bio-fluids, J Mech Med Biol 11 (2011), 1045

[5] T. Hayat, S. Abelman, E. Momoniat and F.M. Mahomed, Magnetic field and endoscope influences on peristaltic transport: An exact solution, Math Comput Appli 15 (2010), 638.

[6] A.A. Khan, R. Ellahi and M. Usman, The effects of variable viscosity on the peristaltic flow of a non-Newtonian fluid through a porous medium in an inclined channel with slip boundary conditions, J Porous Media 16 (2013), 59.

[7] R. Ellahi, A. Riaz and S. Nadeem, Three dimensional peristaltic flow of Williamson fluid in a rectangular duct, Indian $J$ Phys 8 (2013), 1275.

[8] S. Nadeem, A. Riaz and R. Ellahi, Peristaltic flow of a Jeffrey fluid in a rectangular duct having compliant walls, Chem Industry \& Chem Eng Quarterly 19 (2013), 399.

[9] A.A. Khan, R. Ellahi and K. Vafai, Peristaltic transport of a Jeffrey fluid with variable viscosity through a porous medium in an asymmetric channel, Advances in Math Phys 2012 (2012), 169642 (15 pages).

[10] R. Ellahi, A. Riaz, S. Nadeem and M. Mushtaq, Series solutions of magnetohydrodynamic peristaltic flow of a Jeffrey fluid in eccentric cylinders, Appl Math Inf Sci 7 (2013), 1441.

[11] M. Keimanesh, M.M. Rashidi, A.J. Chamkha and R. Jafari, Study of a third grade non-Newtonian fluid flow between two parallel plates using the multi-step differential transform method, Comm Math Appli 62 (2011), 2871.

[12] O. Anwar Bég, M.M. Rashidi, T.A. Bég and M. Asadi, Homotopy analysis of transient magneto-bio-fluid dynamics of micropolar squeeze film in a porous medium: A model for magneto-bio-rheological lubrication, J Mech Med Biol 12 (2012), 1250051 (21 pages).

[13] M.M. Rashidi, M.T. Rastegari, M. Asadi and O. Anwar Bég, A study of non-Newtonian flow and heat transfer over a non- 
isothermal wedge using the homotopy analysis method, Chem Eng Comm 199 (2012), 231.

[14] Kh.S. Mekheimer, The influence of heat transfer and magnetic field on peristaltic transport of a Newtonian fluid in a vertical annulus: Application of an endoscope, Physics Letters A 372 (2008), 1657.

[15] M. Kothandapani and S. Srinivas, On the influence of wall properties in the MHD peristaltic transport with heat transfer and porous medium, Physics Letters A 372 (2008), 4586.

[16] S. Nadeem, N.S. Akbar, T. Hayat and A.A. Hendi, Peristaltic flow of Walter's B fluid in endoscope, Appl Math Mech 32 (2012), 689

[17] S. Nadeem, T. Hayat, N.S. Akbar and M.Y. Malik, On the influence of heat transfer in peristalsis with variable viscosity, Int J Heat and Mass Transfer 52 (2009), 4722.

[18] M. Mustafa, T. Hayat, I. Pop and A. Hendi, Stagnation-point flow and heat transfer of a Casson fluid towards a stretching sheet, Z Naturforsch 6 (2012), 70.

[19] K. Vajravelu, S. Sreenadh and P. Lakshminarayana, The influence of heat transfer on peristaltic transport of a Jeffrey fluid in a vertical porous stratum, Comm Nonlinear Sci Numer Simul 16 (2011), 3107.

[20] D. Tripathi, A mathematical model for swallowing of food bolus through the esophagus under the influence of heat transfer, Int J Thermal Sci 51 (2012), 91.

[21] T. Hayat, S. Hina and A.A. Hendi, Slip effects on peristaltic transport of a Maxwell fluid with heat and mass transfer, $J$ Mech Med Biol 12 (2012), 1250001 (22 pages).

[22] T. Hayat, S. Noreen and A. Alsaedi, The slip and induced magnetic field effects on the peristaltic transport with heat transfer, J Mech Med Biol 12 (2012), 1250068 (15 pages).

[23] T. Hayat and F.M. Abbasi, Peristaltic mechanism in an asymmetric channel with heat transfer, Math Comput Appli 15 (2010), 621.

[24] T. Hayat, N. Saleem and N. Ali, Effect of induced magnetic field on peristaltic transport of a Carreau fluid, Comm Nonlinear Sci Numer Simul 15 (2010), 2407.

[25] R. Ellahi, A. Riaz, S. Nadeem and M. Ali, Peristaltic flow of a Carreau fluid in a rectangular duct through a porous medium, Math Prob Eng 2012 (2012), 329639 (24 pages).

[26] L.M. Srivastava and V.P. Srivastava, Peristaltic transport of a power law fluid: Applications to the ductus efferentes of the reproductive tract, Rheol Acta 27 (1988), 428.

[27] H.S. Lew, Y.C. Fung and C.B. Lowenstein, Peristaltic carrying and mixing of chyme, J Biomech 4 (1971), 297.

[28] O. Eytan and D. Elad, Analysis of intra-uterine fluid motion induced by uterine contractions, Bull Math Biol 61 (1999), 221.

[29] H. Strohmer, A. Obruca, K.M. Rander and W. Feichtinger, Relationship of the individual uterine size and the endometrial thickness in stimulated cycles, Fertil Steril 61 (1994), 972.

\section{Appendix A}

Here we provide the quantities appearing in the flow analysis.

$$
R_{1}=\frac{-2\left(F_{0}+h_{1}-h_{2}\right)}{\left(h_{1}-h_{2}\right)^{3}},
$$

$$
\begin{aligned}
& R_{2}=\frac{3\left(F_{0}+h_{1}-h_{2}\right)\left(h_{1}+h_{2}\right)}{\left(h_{1}-h_{2}\right)^{3}}, \\
& R_{3}=\frac{-h_{1}^{3}-6 F_{0} h_{1} h_{2}-3 h_{1}^{2} h_{2}+3 h_{1} h_{2}^{2}+h_{2}^{3}}{\left(h_{1}-h_{2}\right)^{3}},
\end{aligned}
$$

$R_{4}=$

$$
\begin{aligned}
& \frac{-\left(h_{1}+h_{2}\right)\left(2 h_{1} h_{2}\left(-h_{1}+h_{2}\right)+F_{0}\left(h_{1}^{2}-4 h_{1} h_{2}+h_{2}^{2}\right)\right)}{2\left(h_{1}-h_{2}\right)^{3}}, \\
& B_{1}=B i_{2}+B i_{1}\left(-1+B i_{2}\left(h_{1}-h_{2}\right)\right) \text {, } \\
& B_{2}=3 h_{1}^{2} R_{1}^{2}+3 h_{1} R_{1} R_{2}+R_{2}^{2}, \\
& B_{3}=3 h_{2}^{2} R_{1}^{2}+3 h_{2} R_{1} R_{2}+R_{2}^{2} \text {, } \\
& B_{4}=3\left(h_{1}^{4}-h_{2}^{4}\right) R_{1}^{2}+4\left(h_{1}^{3}-h_{2}^{3}\right) R_{1} R_{2} \\
& +2\left(h_{1}^{2}-h_{2}^{2}\right) R_{2}^{2} \text {, } \\
& B_{5}=1+B i_{2} h_{2} \text {, } \\
& B_{6}=-4 h_{1} B_{2} B_{5} \text {, } \\
& B_{7}=9 h_{2}^{2} R_{1}^{2}+8 h_{2} R_{1} R_{2}+2 R_{2}^{2} \text {, } \\
& B_{8}=B_{3}+h_{2} R_{1} R_{2}+R_{2}^{2} \text {, } \\
& B_{9}=-3\left(h_{1}^{3}-4 h_{2}^{3}\right) R_{1}^{2}-4\left(h_{1}^{2}-3 h_{2}^{2}\right) R_{1} R_{2} \\
& -2\left(h_{1}^{2}-h_{2}^{2}\right) R_{2}^{2} \text {, } \\
& B_{10}=3\left(-h_{1}^{3}+h_{2}^{3}\right) R_{1}^{2}+4\left(-h_{1}^{2}+h_{2}^{2}\right) R_{1} R_{2} \\
& +2\left(-h_{1}+h_{2}\right) R_{2}^{2} \text {, } \\
& A_{1}=-3 B r R_{1}^{2}, \quad A_{2}=-4 B r R_{1} R_{2} \text {, } \\
& A_{3}=-2 B r R_{2}^{2} \text {, } \\
& A_{4}=\frac{1}{B_{1}}\left[4 B i_{2} B r h_{1} B_{2}+B i_{1}\left\{-4 B r h_{2} B_{3}\right.\right. \\
& \left.\left.+B i_{2}\left(1+B r B_{4}\right)\right\}\right] \text {, } \\
& A_{5}=\frac{1}{B_{1}}\left[B r\left\{B_{6}+h_{2}\left(B_{7}+B_{5} B_{8}\right)\right\}\right. \\
& +B i_{1}\left\{-1+B r h_{1} B_{9}\right. \\
& \left.\left.+B i_{2} h_{2}\left(-1+B r h_{1} B_{10}\right)\right\}\right] \text {, }
\end{aligned}
$$$$
M_{1}=-\frac{27}{5}(-1+n) R_{1}^{3} \text {, }
$$$$
M_{2}=-9(-1+n) R_{1}^{2} R_{2} \text {, }
$$$$
M_{3}=-\frac{1}{5\left(h_{1}-h_{2}\right)^{3}}\left[10 F_{1}-9\left(h_{1}-h_{2}\right)^{3}\right.
$$$$
(-1+n) R_{1}^{2}\left(3\left(3 h_{1}^{2}+4 h_{1} h_{2}+3 h_{2}^{2}\right) R_{1}\right.
$$$$
\left.\left.+10\left(h_{1}+h_{2}\right) R_{2}\right)\right) \text {, }
$$ 


$$
\begin{aligned}
& M_{4}=\frac{1}{5\left(h_{1}-h_{2}\right)^{3}}\left[3 \left(5 F_{1}\left(h_{1}+h_{2}\right)\right.\right. \\
& -3\left(h_{1}-h_{2}\right)^{3}(-1+n) R_{1}^{2}\left(6\left(h_{1}+h_{2}\right)\right. \\
& \left(h_{1}^{2}+3 h_{1} h_{2}+h_{2}^{2}\right) R_{1} \\
& \left.\left.\left.+5\left(h_{1}^{2}+4 h_{1} h_{2}+h_{2}^{2}\right) R_{2}\right)\right)\right] \text {, } \\
& M_{5}=-\frac{1}{5\left(h_{1}-h_{2}\right)^{3}}\left[3 h _ { 1 } h _ { 2 } \left(10 F_{1}-3\left(h_{1}-h_{2}\right)^{3}\right.\right. \\
& (-1+n) R_{1}^{2}\left(3\left(4 h_{1}^{2}+7 h_{1} h_{2}+4 h_{2}^{2}\right) R_{1}\right. \\
& \left.\left.\left.+10\left(h_{1}+h_{2}\right) R_{2}\right)\right)\right] \text {, } \\
& M_{6}=-\frac{1}{10\left(h_{1}-h_{2}\right)^{3}}\left[5 F _ { 1 } ( h _ { 1 } + h _ { 2 } ) \left(h_{1}^{2}\right.\right. \\
& \left.-4 h_{1} h_{2}+h_{2}^{2}\right)+18 h_{1}^{2}\left(h_{1}-h_{2}\right)^{3} h_{2}^{2} \\
& \left.(-1+n) R_{1}^{2}\left(6\left(h_{1}+h_{2}\right) R_{1}+5 R_{2}\right)\right] \text {, } \\
& L_{1}=-\frac{4}{5} B r R_{1}\left(10 M_{1}+27(-1+n) R_{1}^{3}\right) \text {, } \\
& L_{2}=-\frac{4}{5} \operatorname{Br}\left(5 M_{1} R_{2}+9 R_{1}\left(M_{2}\right.\right. \\
& \left.\left.+6(-1+n) R_{1}^{2} R_{2}\right)\right), \\
& L_{3}=-2 B r\left(3 M_{3} R_{1}+2 R_{2}\left(M_{2}\right.\right. \\
& \left.\left.+9(-1+n) R_{1}^{2} R_{2}\right)\right) \text {, } \\
& L_{4}=-4 B r\left(M_{4} R_{1}+M_{3} R_{2}+4(-1+n) R_{1} R_{2}^{3}\right. \text {, } \\
& L_{5}=-4 B r R_{2}\left(M_{4}+(-1+n) R_{2}^{3}\right) \text {, } \\
& L_{6}=\frac{2 B r}{5 B_{1}}\left[-2 B i_{1} h_{2}\left\{6 h _ { 2 } ^ { 4 } R _ { 1 } \left(10 M_{1}\right.\right.\right. \\
& \left.+27(-1+n) R_{1}^{3}\right)+10 R_{2}\left(M_{4}\right. \\
& \left.+(-1+n) R_{2}^{3}\right)+15 h_{2}\left(M_{4} R_{1}+M_{3} R_{2}\right. \\
& \left.+4(-1+n) R_{1} R_{2}^{3}\right)+5 h_{2}^{3}\left(5 M_{1} R_{2}\right. \\
& \left.+9 R_{1}\left(M_{2}+6(-1+n) R_{1}^{2} R_{2}\right)\right) \\
& +10 h_{2}^{2}\left(3 M_{3} R_{1}+2 R_{2}\left(M_{2}\right.\right. \\
& \left.\left.\left.+9(-1+n) R_{1}^{2} R_{2}\right)\right)\right\} \\
& +B i_{2}\left\{2 B i_{1} h_{1}^{6} R_{1}\left(10 M_{1}+27(-1+n) R_{1}^{3}\right)\right. \\
& +20 h_{1} R_{2}\left(M_{4}+(-1+n) R_{2}^{3}\right) \\
& +10 h_{1}^{2}\left(3 M_{3} R_{2}+M_{4}\left(3 R_{1}+B i_{1} R_{2}\right)\right. \\
& \left.+(-1+n) R_{2}^{3}\left(12 R_{1}+B i_{1} R_{2}\right)\right) \\
& +5 h_{1}^{4}\left(2\left(5 M_{1}+54(-1+n) R_{1}^{3}\right) R_{2}\right.
\end{aligned}
$$

$$
\begin{aligned}
& +2 M_{2}\left(9 R_{1}+B i_{1} R_{2}\right)+3 B i_{1} R_{1}\left(M_{3}\right. \\
& \left.\left.+6(-1+n) R_{1} R_{2}^{2}\right)\right) \\
& +10 h_{1}^{3}\left(M_{3}\left(6 R_{1}+B i_{1} R_{2}\right)\right. \\
& +4 R_{2}\left(M_{2}+9(-1+n) R_{1}^{2} R_{2}\right) \\
& \left.+B i_{1} R_{1}\left(M_{4}+4(-1+n) R_{2}^{3}\right)\right) \\
& +2 h_{1}^{5}\left(5 M_{1}\left(12 R_{1}+B i_{1} R_{2}\right)\right. \\
& +9\left(18(-1+n) R_{1}^{4}+B i_{1} R_{1}\left(M_{2}\right.\right. \\
& \left.\left.\left.+6(-1+n) R_{1}^{2} R_{2}\right)\right)\right\}-B i_{1} h_{2}^{2}\left(h _ { 2 } ^ { 4 } \left(20 M_{1} R_{1}\right.\right. \\
& \left.+54(-1+n) R_{1}^{4}\right)+10 R_{2}\left(M_{4}\right. \\
& \left.+(-1+n) R_{2}^{3}\right)+10 h_{2}\left(M_{4} R_{1}+M_{3} R_{2}\right. \\
& \left.+4(-1+n) R_{1} R_{2}^{3}\right)+2 h_{2}^{3}\left(5 M_{1} R_{2}\right. \\
& \left.+9 R_{1}\left(M_{2}+6(-1+n) R_{1}^{2} R_{2}\right)\right) \\
& +5 h_{2}^{2}\left(3 M_{3} R_{1}+2 R_{2}\left(M_{2}\right.\right. \\
& \left.\left.\left.\left.\left.+9(-1+n) R_{1}^{2} R_{2}\right)\right)\right)\right\}\right] \text {, } \\
& L_{7}=\frac{2 B r}{5 B_{1}}\left[2 B i _ { 1 } h _ { 1 } ^ { 6 } ( 1 + B i _ { 2 } h _ { 2 } ) R _ { 1 } \left(10 M_{1}\right.\right. \\
& \left.+27(-1+n) R_{1}^{3}\right)+10 h_{1}^{2}\left(1+B i_{2} h_{2}\right)\left(3 M_{3} R_{2}\right. \\
& +M_{4}\left(3 R_{1}+B i_{1} R_{2}\right)+(-1+n) R_{2}^{3}\left(12 R_{1}\right. \\
& \left.\left.+B i_{1} R_{2}\right)\right)+5 h_{1}^{4}\left(1+B i_{2} h_{2}\right)\left(2 \left(5 M_{1}\right.\right. \\
& \left.+54(-1+n) R_{1}^{3}\right) R_{2}+2 M_{2}\left(9 R_{1}+B i_{1} R_{2}\right) \\
& \left.+3 B i_{1} R_{1}\left(M_{3}+6(-1+n) R_{1} R_{2}^{2}\right)\right) \\
& +10 h_{1}^{3}\left(1+B i_{2} h_{2}\right)\left(M_{3}\left(6 R_{1}+B i_{1} R_{2}\right)\right. \\
& +4 R_{2}\left(M_{2}+9(-1+n) R_{1}^{2} R_{2}\right) \\
& \left.+B i_{1} R_{1}\left(M_{4}+4(-1+n) R_{2}^{3}\right)\right) \\
& +2 h_{1}^{5}\left(1+B i_{2} h_{2}\right)\left(5 M_{1}\left(12 R_{1}+B i_{1} R_{2}\right)\right. \\
& +9\left(18(-1+n) R_{1}^{4}\right. \\
& \left.\left.+B i_{1} R_{1}\left(M_{2}+6(-1+n) R_{1}^{2} R_{2}\right)\right)\right) \\
& -h_{2}\left\{2 B i_{2} h_{2}^{5} R_{1}\left(10 M_{1}+27(-1+n) R_{1}^{3}\right)\right. \\
& +20 R_{2}\left(M_{4}+(-1+n) R_{2}^{3}\right) \\
& +10 h_{2}\left(3 M_{3} R_{2}+M_{4}\left(3 R_{1}+B i_{2} R_{2}\right)\right. \\
& \left.+(-1+n) R_{2}^{3}\left(12 R_{1}+B i_{2} R_{2}\right)\right) \\
& +5 h_{2}^{3}\left(2\left(5 M_{1}+54(-1+n) R_{1}^{3}\right) R_{2}\right. \\
& +2 M_{2}\left(9 R_{1}+B i_{2} R_{2}\right) \\
& \left.+3 B i_{2} R_{1}\left(M_{3}+6(-1+n) R_{1} R_{2}^{2}\right)\right)
\end{aligned}
$$




$$
\begin{aligned}
& +10 h_{2}^{2}\left(M_{3}\left(6 R_{1}+B i_{2} R_{2}\right)\right. \\
& +4 R_{2}\left(M_{2}+9(-1+n) R_{1}^{2} R_{2}\right) \\
& \left.+B i_{2} R_{1}\left(M_{4}+4(-1+n) R_{2}^{3}\right)\right) \\
& +2 h_{2}^{4} \times\left(5 M_{1}\left(12 R_{1}+B i_{2} R_{2}\right)\right. \\
& +9\left(18(-1+n) R_{1}^{4}\right. \\
& \left.\left.\left.+B i_{2} R_{1}\left(M_{2}+6(-1+n) R_{1}^{2} R_{2}\right)\right)\right)\right\} \\
& -h_{1}\left\{-20\left(1+B i_{2} h_{2}\right) R_{2}\left(M_{4}+(-1+n) R_{2}^{3}\right)\right. \\
& +B i_{1} h_{2}\left(2 B i_{2} h_{2}^{5} R_{1}\left(10 M_{1}+27(-1+n) R_{1}^{3}\right)\right. \\
& +20 R_{2}\left(M_{4}+(-1+n) R_{2}^{3}\right) \\
& +10 h_{2}\left(3 M_{3} R_{2}+M_{4}\left(3 R_{1}+B i_{2} R_{2}\right)\right.
\end{aligned}
$$

$$
\begin{aligned}
& \left.+(-1+n) R_{2}^{3}\left(12 R_{1}+B i_{2} R_{2}\right)\right) \\
& +5 h_{2}^{3}\left(2\left(5 M_{1}+54(-1+n) R_{1}^{3}\right) R_{2}\right. \\
& +2 M_{2}\left(9 R_{1}+B i_{2} R_{2}\right) \\
& \left.+3 B i_{2} R_{1}\left(M_{3}+6(-1+n) R_{1} R_{2}^{2}\right)\right) \\
& +10 h_{2}^{2}\left(M_{3}\left(6 R_{1}+B i_{2} R_{2}\right)\right. \\
& +4 R_{2}\left(M_{2}+9(-1+n) R_{1}^{2} R_{2}\right) \\
& \left.+B i_{2} R_{1}\left(M_{4}+4(-1+n) R_{2}^{3}\right)\right) \\
& +2 h_{2}^{4}\left(5 M_{1}\left(12 R_{1}+B i_{2} R_{2}\right)\right. \\
& +9\left(18(-1+n) R_{1}^{4}\right. \\
& \left.\left.\left.\left.\left.+B i_{2} R_{1}\left(M_{2}+6(-1+n) R_{1}^{2} R_{2}\right)\right)\right)\right)\right\}\right] .
\end{aligned}
$$



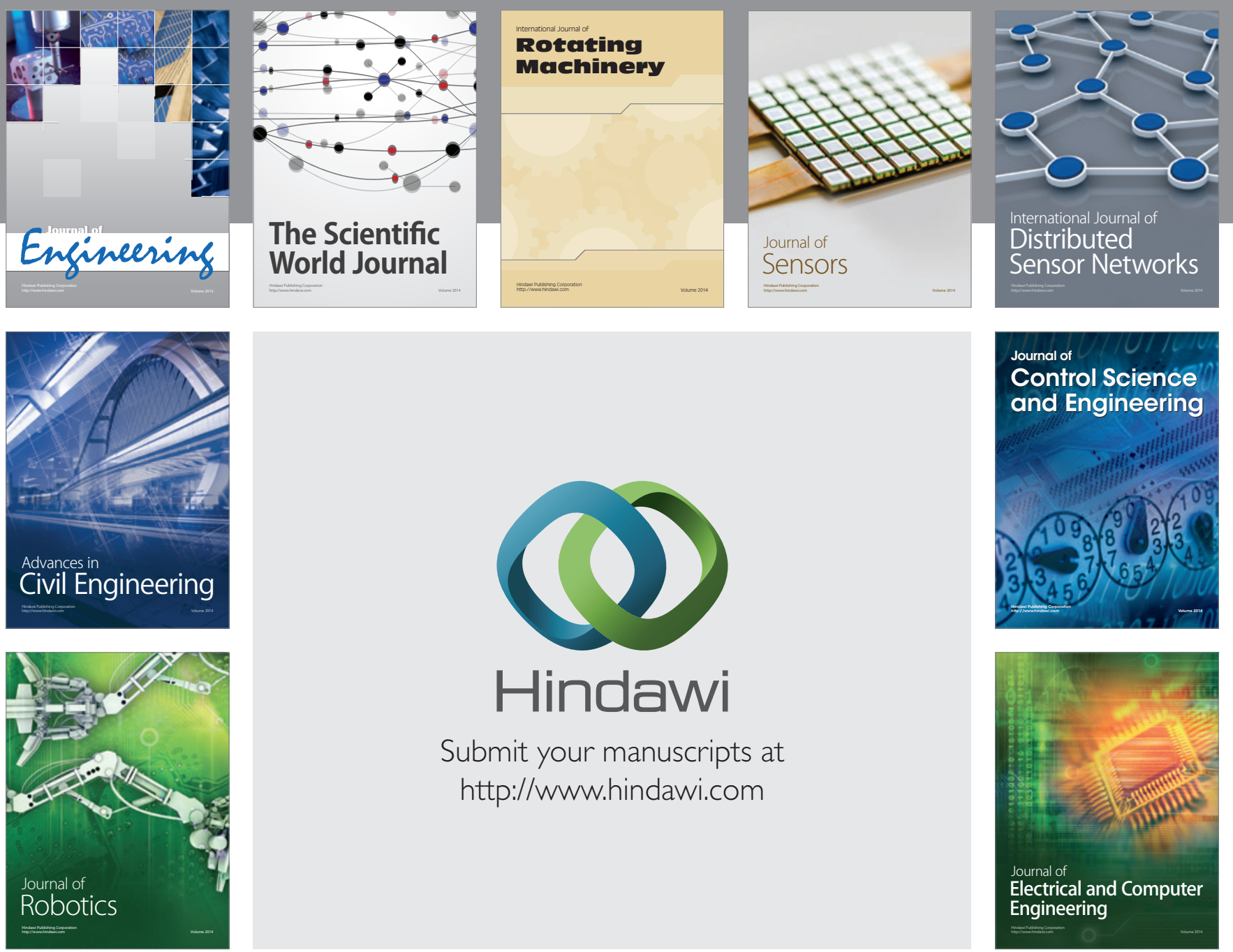

Submit your manuscripts at

http://www.hindawi.com
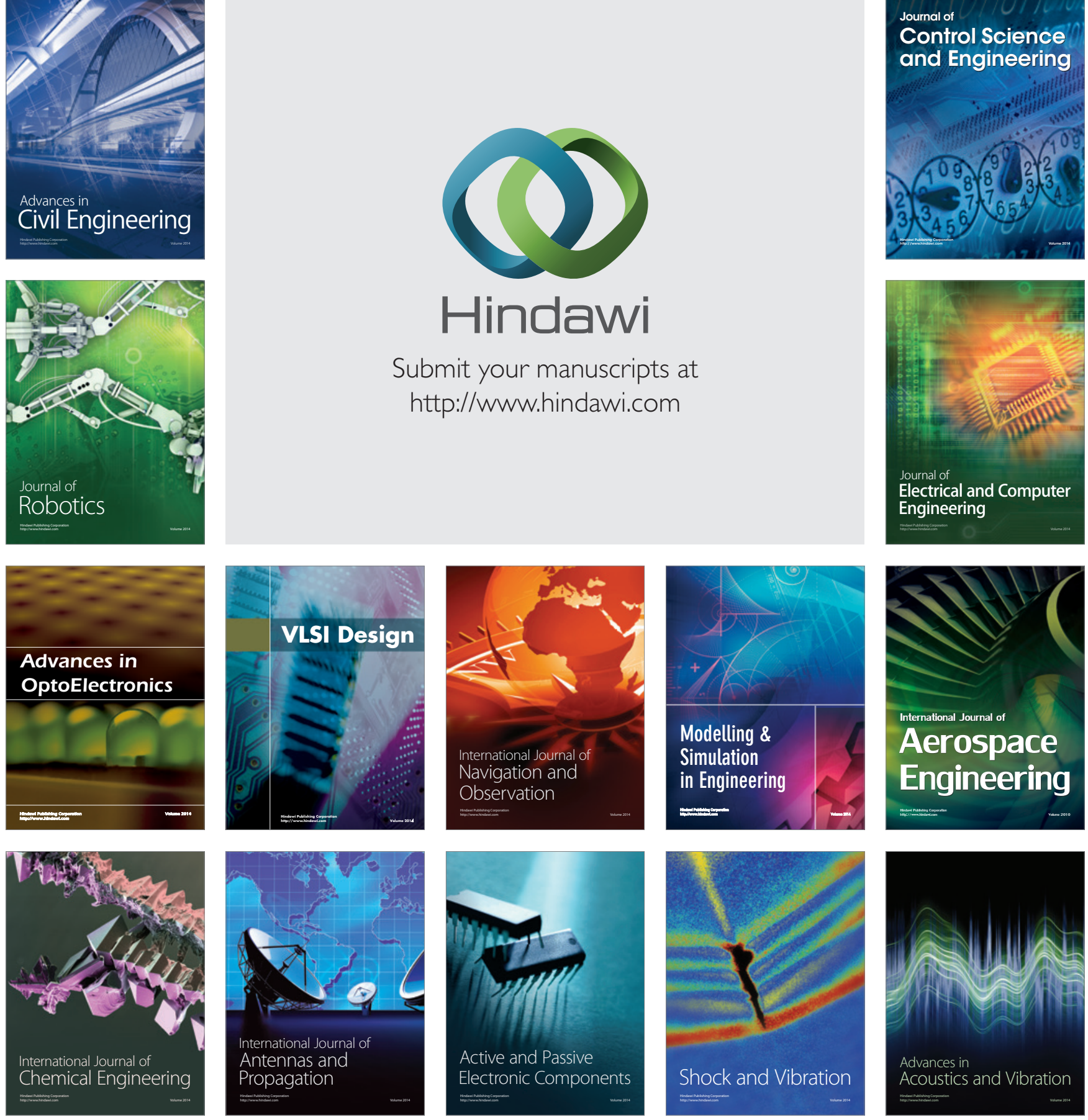